\section{Segunda encuesta antropometrica de embarazadas de la ciudad de Buenos Aires, Argentina 2010}

\section{The second anthropometric survey of pregnant women in the city of Buenos Aires, Argentina 2010}

Andrés Bolzán 1

Silvina Dupraz 2

Laura Piaggio 3

Marina Rolón 4

Macedra Guadalupe 5

1 Dirección General de Redes de Salud. Ministerio de Salud de la Ciudad Autónoma de Buenos Aires. Av. Almacio Alcorta y Monasterio. CABA, Argentina. E-mail andresbolzan@yahoo.com.ar

2-5 Programa Nutricional (DGPC - MS - GCBA). Htal. Gral de Niños «Pedro de Elizalde». Montes de Oca $40-$ Pab. Cardiología $-5^{\circ}$ piso.

\begin{abstract}
Objectives: to present the results of the second anthropometric survey of pregnant women in Buenos Aires, Argentina.

Methods: measurements were taken of all pregnant women attending the outpatient services of 12 public maternity hospitals during one week. Measurements included weight, height and body mass index (BMI). The survey was a structured survey.

Results: the study covered 2811 pregnant women born in Argentina (49\%) and other countries (51\%). Of the latter, $49 \%$ were women born in Bolivia (22\%), Paraguay (19\%) and Peru (8\%). The main nutritional problem was overweight and there were statistically significant differences according to country of birth. The BMI curves for pregnant adults and pregnant adolescents exhibited clear differences.

Conclusions: the survey provided a profile of the nutritional status of pregnant women, using national BMI growth curves adjusted for gestational age and enabled observation of a number of determining social factors.
\end{abstract}

Key words Nutritional status, Anthropometry Pregnancy

\section{Resumen}

Objetivos: mostrar los resultados de la segunda encuesta antropométrica en embarazadas en Buenos Aires, Argentina.

Métodos: fueron medidas todas las embarazadas que concurrieron a control de salud durante una semana en los consultorios externos y centros de salud de las 12 maternidades públicas valorándose su peso, talla e indice de masa corporal (IMC). Se empleo una encuesta estructurada.

Resultados: fueron evaluadas 2811 embarazadas, nacidas en Argentina (49\%) y en otros paises (51\%). Dentro de este segundo grupo, un 49\% correspondió a mujeres nacidas en Bolivia (22\%), Paraguay (19\%) y Perú (8\%). El problema nutricional más importante lo constituyó el exceso de peso con diferencias estadisticamente significativas según pais de nacimiento. Se observaron dos curvas claramente diferenciadas en el IMC de embarazadas adolescentes $y$ adultas.

Conclusiones: la investigación permitió obtener el perfil de estado nutricional durante la gestación empleando curvas nacionales de incremento del IMC ajustado por edad gestacional y observar el efecto de algunos determinantes sociales.

Palabras clave Estado nutricional, Antropometría, Embarazadas 


\section{Introduccion}

El estado nutricional materno tiene un efecto determinante sobre el crecimiento fetal, el peso del recién nacido, el tamaño de la placenta y la morbimortalidad feto neonatal.1,2 estando demostrada su asociación con las tasas de bajo peso al nacer. Por consiguiente, la determinación en una comunidad de la prevalencia de diferentes niveles de estado nutricional durante el embarazo es una actividad de prevención primaria esencial. Por otra parte, cuando las tasas de mortalidad en un país se van reduciendo, llegan a ser un indicador con poca sensibilidad para estimar el estado de salud y en cambio adquieren importancia otros indicadores relacionados a las formas de vida, el acceso a servicios básicos y a la alimentación.

El estado nutricional materno se puede determinar de diversas maneras. A los fines prácticos de asistencia la evaluación antropométrica resulta en una metodología sencilla, de bajo costo y sensible. 3 Se ha demostrado que el estado nutricional tiene relación con el peso al nacer. En los últimos años se han considerado el índice de masa corporal (IMC) como un indicador de estado nutricional durante la gestación. El IMC ha sido adoptado en la década de los 80 como un posible indicador de estado nutricional asumiendo como criterio el máximo coeficiente de correlación de Pearson para el peso $(\mathrm{r}=0.86)$ junto con el mínimo coeficiente para la talla $(r=0.04)$. De esa forma se controla la variable de mayor componente genético del tamaño corporal. El IMC materno se asocia con la prematurez, el bajo peso al nacer, el retardo de crecimiento intrauterino, el peso placentario y se emplea como predictor del tamaño al nacer neonatal.4-6

En la Ciudad de Buenos Aires, la mayor de la Argentina, existen doce maternidades públicas en donde se producen unos 30 mil nacimientos anuales. Además, cada maternidad presenta centros periféricos de salud que asisten el embarazo en diferentes barrios. La Ciudad de Buenos Aires presenta la menor tasa de mortalidad infantil del país, sin embargo, hay menos información sobre el estado nutricional de las madres cuyos partos son asistidos en la Ciudad.

Por otra parte, la Argentina ha desarrollado sus curvas de IMC ajustadas por edad gestacional para evaluar el estado nutricional materno en todo el país. ${ }^{7}$

El objetivo del presente trabajo es presentar el estado nutricional de las embarazadas de la Ciudad de Buenos Aires 2010 empleando las curvas de referencia nacionales de IMC ajustadas por edad gestacional.

\section{Metodos}

La Ciudad de Buenos Aires, Argentina,tiene una población de casi 3 millones de habitantes y la cantidad de nacimientos anuales ronda los $45 \mathrm{mil}$, incluyendo los nacimientos públicos y privados siendo que el subsector público de salud atiende unos 30 mil partos distribuidos en 12 maternidades. Periódicamente se realiza un censo semanal de las embarazadas que se asisten en todos los efectores de salud de la Ciudad. Durante el año 2010 fue realizada la segunda encuesta antropométrica de embarazadas, siendo un censo de una semana de duración (26-30 Abril 2010) de todas las gestantes que concurrieron al control a los hospitales y centros de salud de Ciudad de Buenos Aires (CESAC).

Se empleó una encuesta estructurada, aplicada en los CESAC y a los servicios de obstetricia de hospitales de la Ciudad.

Se consideraron las siguientes variables: efector de salud, edad, edad gestacional, país de nacimiento, lugar de residencia, estudios, número de embarazos previos, antecedente de recién nacido de bajo peso, peso previo al embarazo, peso en la consulta, talla, suplementación con hierro y ácido fólico.

El IMC fue calculado (IMC (Q) = peso / talla 2$)$ y posteriormente los datos crudos fueron convertidos a escore $Z$, aplicando la fórmula: $Z=(Q-M) L-1 /$ $\mathrm{LS}$, donde $\mathrm{Q}=\mathrm{IMC}$ de la embarazada, $\mathrm{L}=$ valor de la transformación de Box-Cox para la kurtosis, $\mathrm{S}=$ coeficiente de variación, $\mathrm{M}=$ mediana según edad gestacional del IMC.

Para el cálculo estadístico se empleó un programa en Stata 9.0. Se realizaron análisis de varianza para las variables continuas, testeando previamente la homocedasticidad de las variables mediante el test de Bartlett y cuando fuera posible, el test post hoc de Bonferroni. Las variables nominales o categóricas se testearon mediante el $\chi^{2}$. El IMC fue categorizado en los puntos de corte basados en la normativa nacional para evaluar estado nutricional durante la gestación aplicando los escore $\mathrm{Z}$ ajustados para la edad gestacional (IMC/EG): bajo= IMC/EG $<-1$; adecuado $=\mathrm{IMC} / \mathrm{EG} \geq-1 \mathrm{y} \leq+1$; sobrepeso $=$ $\mathrm{IMC} / \mathrm{EG}>+1$ D.E. $\mathrm{y} \leq+2$; obesidad $=\mathrm{IMC} / \mathrm{EG}>+2$.

\section{Resultados}

La Tabla 1 muestra la distribución porcentual del IMC según maternidad y sectores del área bajo la curva, la Tabla 2 indica la interpretación del estado nutricional según el IMC por maternidad y Región sanitaria, la Tabla 3 y 4 presenta los indicadores de peso, talla e IMC previos al embarazo y los valores 
porcentuales de distribución según nacionalidad de la madre, en tanto la Tabla 5 muestra la distribución del IMC en relación a la edad gestacional según grupo de edad materna. Fueron evaluadas 2811 embarazadas. La población estudiada se dividió en dos partes prácticamente iguales según el país de nacimiento: nacidas en Argentina (49\%) y en otros países (51\%). Dentro de este segundo grupo, un 49\% correspondió a mujeres nacidas en Bolivia (22\%), Paraguay (19\%) y Perú (8\%). Entre las embarazadas adolescentes, el porcentaje de mujeres nacidas en Argentina fue del $65 \%$, y el $35 \%$ restante nacidas en los mencionados países de Latinoamérica. La edad media de las embarazadas fue de 26 años. Las adolescentes representan un $11 \%$ de la muestra. Casi el $40 \%$ de las encuestadas son primigestas, en tanto un $9 \%$ son grandes multígestas, de cuatro y más embarazos previos. Entre las adolescentes, un $84 \%$ son primigestas y un $13 \%$ han tenido un embarazo previo. El problema nutricional más importante, a partir de la evaluación antropométrica, lo constituye el exceso de peso (alto IMC para la edad gestacional). El bajo peso, que se considera con un punto de corte muy sensible (<-1.0 DE) debido a la vulnerabilidad biológica del período gestacional, se encuentra por debajo de lo esperable en una distribución poblacional normal. No se registraron diferencias significativas entre las regiones sanitarias $\left(\chi^{2}=2.1 ; p=0.90\right)$. Se observaron diferencias estadísticamente significativas en la talla $(\mathrm{F}=113.8$; $p=0.001)$ y en el peso previo $(\mathrm{F}=13.9 ; p=0.001)$ respecto del factor país de nacimiento. No ocurrió lo mismo para el índice de masa corporal previo al embarazo. La distribución de sectores bajo el área de la curva de IMC según el país de nacimiento mostró diferencias estadísticamente significativas $\left(\chi^{2}=35.4\right.$; $p=0.001)$ en los porcentajes del indicador según los puntos de corte establecidos. Se puede observar que, si consideramos el sector por debajo de -1 desvío estándar de la referencia, las embarazadas argentinas presentan el mayor componente de bajo peso $(10.3 \%)$, seguido de las peruanas $(8.7 \%)$, las paraguayas $(6.4 \%)$ y finalmente las bolivianas (5.9\%). En tanto, para el sobrepeso y obesidad (>+1 desvío estándar) las argentinas registraron el mayor porcentaje $(27.4 \%)$, seguido de las paraguayas $(24.4 \%)$, las bolivianas $(24.2 \%)$ y las peruanas $(21.9 \%)$. En ningún caso el bajo peso materno superó el límite probabilísticamente esperado, siendo que por debajo de - 1 desvío estándar no hubo para ningún grupo sectores mayores a $15,8 \%$. Se puede observar que la mayor prevalencia de sobrepeso y obesidad se encuentra en los niveles educativos más bajos: 1 y 2 , con sesgo a derecha de la distribución. Al tomar los sectores considerados como bajo peso (debajo de -2 DE y - 1 DE), se observa mayor porcentaje de embarazadas en los niveles educativos más altos: 3 y 4 , y, a la inversa, el porcentaje de exceso de peso $(>+1 \mathrm{DE} y+2 \mathrm{DE})$ es menor en este grupo. Hubo diferencias estadísticamente significativas en las curvas de IMC según el nivel educativo materno (Figura 1). Estas diferencias son adjudicables a los niveles educativos 3 y 4 , donde las medias de IMC son menores a las de los niveles 1 y 2 . El grupo de adolescentes comienza con un IMC 3 puntos menor (en promedio) al del grupo de las adultas y, al final del embarazo, tienen un IMC 6 puntos mayor al de inicio; mientras que entre las adultas este "incremento" es de 4 puntos. En ningún momento del embarazo se cruzan estas curvas, verificándose diferencias estadísticamente significativas entre ambos grupos. Se observan dos curvas claramente diferenciadas en el IMC de embarazadas adolescentes y adultas.

Tabla 1

Distribución porcentual del índice de masa corporal según intervalos bajo el área de la curva del escore z según hospital. Ciudad de Buenos Aires, 2010.

\begin{tabular}{lrcccccc}
\hline \multirow{2}{*}{ Hospital } & $\mathbf{N}$ & \multicolumn{5}{c}{ Sector del área bajo la curva (\%) } \\
\cline { 3 - 7 } & & $<-2$ & $\geq-2.0<-1.0$ & $\geq-1.0 \leq+1.0$ & $>1.0 \leq+2.0$ & $>+2$ & Total \\
\hline Alvarez & 139 & 0.0 & 10.2 & 65.7 & 23.4 & 0.7 & 100.0 \\
Argerich & 42 & 0.0 & 2.4 & 71.4 & 23.8 & 2.4 & 100.0 \\
Durand & 225 & 2.3 & 8.5 & 64.8 & 22.5 & 1.9 & 100.0 \\
Fernandez & 304 & 1.0 & 10.0 & 72.0 & 16.0 & 1 & 100.0 \\
Penna & 173 & 0.6 & 7.6 & 64.3 & 23.4 & 4.1 & 100.0 \\
Piñero & 394 & 1.0 & 7.0 & 65.2 & 23.5 & 3.3 & 100.0 \\
Pirovano & 171 & 1.2 & 7.1 & 67.9 & 19.0 & 4.8 & 100.0 \\
Ramos mejía & 19 & 5.3 & 0.0 & 73.7 & 15.8 & 5.3 & 100.0 \\
Rivadavia & 199 & 1.0 & 3.6 & 67.2 & 25.6 & 2.6 & 100.0 \\
Santojanni & 688 & 1.3 & 7.5 & 63.7 & 22.3 & 5.2 & 100.0 \\
Sarda & 289 & 1.1 & 6.0 & 63.1 & 23.0 & 6.7 & 100.0 \\
Velez Sarfield & 168 & 2.4 & 8.4 & 69.5 & 17.4 & 2.4 & 100.0 \\
Total & 2811 & 1.2 & 7.4 & 66.0 & 21.7 & 3.7 & 100.0 \\
\hline
\end{tabular}


Tabla 2

Distribución en \% del índice de masa corporal según puntos de corte por Región y hospital. Encuesta antropométrica a embarazadas. Ciudad de Buenos Aires 2010.

\begin{tabular}{|c|c|c|c|c|}
\hline \multirow[t]{2}{*}{ Región Sanitaria } & \multirow[t]{2}{*}{ Maternidad } & \multicolumn{3}{|c|}{ Índice de massa corporal* } \\
\hline & & Bajo & Normal & Alto \\
\hline \multirow[t]{5}{*}{ I } & Sarda & 7.1 & 63.1 & 29.8 \\
\hline & Argerich & 2.4 & 71.4 & 26.2 \\
\hline & Penna & 8.2 & 64.3 & 27.5 \\
\hline & Ramos mejia & 5.3 & 73.7 & 21.0 \\
\hline & Total región & 5,7 & 68.0 & 26.0 \\
\hline \multirow[t]{4}{*}{ II } & Santojanni & 8.8 & 63.7 & 27.5 \\
\hline & Piñero & 8.0 & 65.2 & 26.8 \\
\hline & Alvarez & 10.2 & 65.7 & 24.1 \\
\hline & Total región & 9.0 & 65.0 & 26.0 \\
\hline \multirow[t]{3}{*}{ III } & Durand & 10.8 & 64.8 & 24.4 \\
\hline & Velez sarfield & 10.8 & 69.5 & 19.7 \\
\hline & Total región & 10.8 & 67.0 & 22.0 \\
\hline \multirow[t]{4}{*}{ IV } & Fernandez & 11.0 & 72.0 & 17.0 \\
\hline & Pirovano & 8.3 & 67.5 & 24.2 \\
\hline & Rivadavia & 4.6 & 67.2 & 28.2 \\
\hline & Total región & 8.0 & 69.0 & 23.0 \\
\hline$\%$ ponderado & & 8.6 & 66.0 & 25.4 \\
\hline
\end{tabular}

* Bajo: <-1.0 desvio estandar; Normo-peso: entre +/- 1 desvio estandar; Alto: >+1 desvio estandar; Comparaciones: $\chi^{2}=2.1 ; p=0.90$

Tabla 3

Distribución de los indicadores antropométricos maternos al comienzo del embarazo según país de nacimiento. Encuesta antropométrica a embarazadas, Ciudad Autónoma de Buenos Aires 2010.

\begin{tabular}{|c|c|c|c|c|}
\hline \multirow[t]{2}{*}{ Pais de nacimiento } & \multirow[t]{2}{*}{$\mathbf{N}$} & \multirow{2}{*}{$\begin{array}{c}\text { Talla (cm) } \\
(\bar{X} \pm D E)\end{array}$} & \multirow{2}{*}{$\begin{array}{c}\text { Peso previo (kg) } \\
(\bar{X} \pm D E)\end{array}$} & \multirow{2}{*}{$\begin{array}{r}\text { IMC previo } \\
(\bar{X} \pm D E)\end{array}$} \\
\hline & & & & \\
\hline Argentina & 1354 & $159.0 \pm 6.5$ & $59.0 \pm 13.5$ & $23.3 \pm 5.1$ \\
\hline Bolivia & 610 & $153.0 \pm 6.1$ & $56.0 \pm 9.6$ & $23.7 \pm 3.9$ \\
\hline Paraguay & 515 & $158.0 \pm 5.5$ & $59.0 \pm 10.9$ & $23.4 \pm 3.9$ \\
\hline Peru & 229 & $155.0 \pm 5.6$ & $56.0 \pm 10.2$ & $23.7 \pm 4.1$ \\
\hline
\end{tabular}

IMC= índice de masa corporal.

\section{Tabla 4}

Distribución de categorías de índice de masa corporal según país de nacimiento. Encuesta antropométrica a embarazadas, Ciudad Autónoma de Buenos Aires 2010.

\begin{tabular}{|c|c|c|c|c|c|c|c|c|c|c|c|c|}
\hline \multirow[t]{3}{*}{ Pais de nacimiento } & \multicolumn{12}{|c|}{ Sector del área bajo la curva } \\
\hline & \multicolumn{2}{|c|}{$<-2$} & \multicolumn{2}{|c|}{$\geq-2.0<-1.0$} & \multicolumn{2}{|c|}{$\geq-1.0 \leq+1.0$} & \multicolumn{2}{|c|}{$>1.0 \leq+2.0$} & \multicolumn{2}{|c|}{$>+2$} & \multicolumn{2}{|c|}{ Total } \\
\hline & $n$ & $\%$ & $\mathrm{n}$ & $\%$ & $\mathrm{n}$ & $\%$ & $n$ & $\%$ & $\mathrm{n}$ & $\%$ & $\mathrm{n}$ & $\%$ \\
\hline Argentina & 24 & 1,8 & 115 & 8,5 & 845 & 62,4 & 300 & 22,2 & 70 & 5,2 & 1354 & 100,0 \\
\hline Bolivia & 3 & 0,5 & 33 & 5,4 & 426 & 69,8 & 135 & 22,1 & 13 & 2,1 & 610 & 100,0 \\
\hline Paraguay & 3 & 0,6 & 30 & 5,8 & 356 & 69,1 & 112 & 21,7 & 14 & 2,7 & 515 & 100,0 \\
\hline Peru & 4 & 1,7 & 16 & 7,0 & 159 & 69,4 & 45 & 19,7 & 5 & 2,2 & 229 & 100,0 \\
\hline
\end{tabular}


Tabla 5

Distribución del índice de masa corporal según edad materna y edad gestacional por cuatrisemanas. Encuesta antropométrica a embarazadas, Ciudad Autónoma de Buenos Aires 2010.

\begin{tabular}{|c|c|c|c|c|}
\hline $\begin{array}{l}\text { Edad gestacional } \\
\text { (semanas) }\end{array}$ & Grupo & $\mathbf{N}$ & $\bar{X} \pm D E$ & $\begin{array}{c}\text { Intervalo de confianza para } \\
\text { la media al } 95 \%\end{array}$ \\
\hline \multirow[t]{2}{*}{$0-13$} & adolescente & 20 & $22.1 \pm 2.5$ & $21.0-23.3$ \\
\hline & adulta & 146 & $25.6 \pm 4.5$ & $24.8-26.3$ \\
\hline \multirow[t]{2}{*}{$14-17$} & adolescente & 25 & $25.4 \pm 4.3$ & $22.6-26.2$ \\
\hline & adulta & 203 & $25.5 \pm 5.2$ & $24.7-26.2$ \\
\hline \multirow[t]{2}{*}{$18-21$} & adolescente & 29 & $23.2 \pm 3.4$ & $24.1-26.7$ \\
\hline & adulta & 242 & $26.5 \pm 5.3$ & $25.8-27.2$ \\
\hline \multirow[t]{2}{*}{$22-25$} & adolescente & 42 & $24.6 \pm 4.0$ & $23.3-25.8$ \\
\hline & adulta & 282 & $26.9 \pm 5.6$ & $26.3-27.6$ \\
\hline \multirow[t]{2}{*}{$26-29$} & adolescente & 39 & $23.2 \pm 6.0$ & $21.2-25.1$ \\
\hline & adulta & 302 & $27.8 \pm 4.4$ & $27.3-28.3$ \\
\hline \multirow[t]{2}{*}{$30-33$} & adolescente & 56 & $27.0 \pm 4.0$ & $25.9-28.1$ \\
\hline & adulta & 387 & $28.6 \pm 5.2$ & $28.1-29.1$ \\
\hline \multirow[t]{2}{*}{$34-37$} & adolescente & 48 & $28.3 \pm 4.4$ & $27.0-29.6$ \\
\hline & adulta & 514 & $29.1 \pm 5.2$ & $28.7-29.6$ \\
\hline \multirow[t]{2}{*}{$38-41$} & adolescente & 34 & $27.9 \pm 3.1$ & $26.8-29.0$ \\
\hline & adulta & 308 & $29.4 \pm 5.7$ & $28.8-30.1$ \\
\hline
\end{tabular}

$\mathrm{F}=67.6 ; p<0.001$

Figura 1

Media e intervalos de confianza al 95\% del índice de masa corporal según nivel educativo. Encuesta antropométrica a embarazadas, Ciudad Autónoma de Buenos Aires 2010.

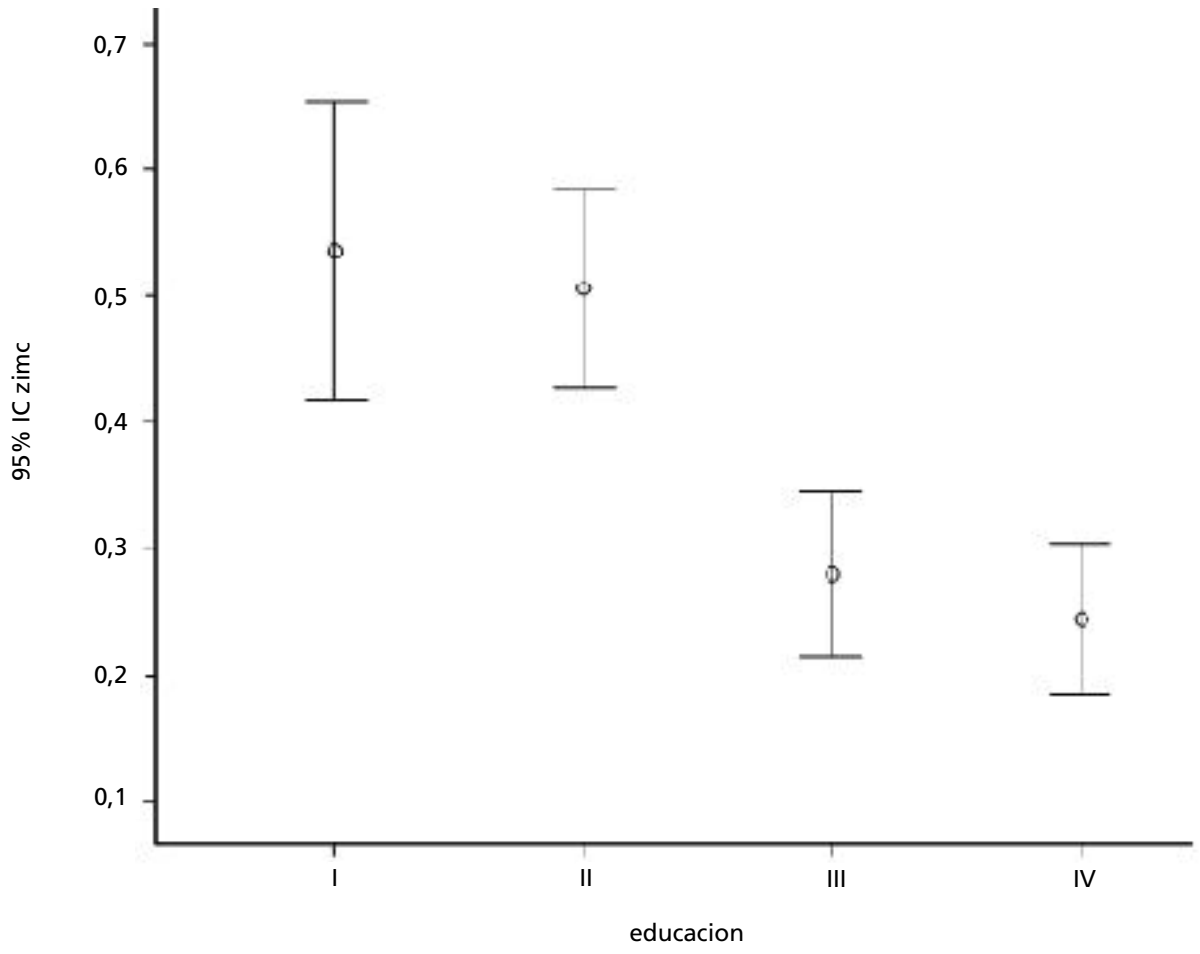

$\mathrm{I}=$ hasta primario incompleto; II= primario completo; III= secundario incompleto; IV= secundario completo y más; $\mathrm{F}=12.4 p<0.001$. I vs III $(p=0.002)$; I vs IV $(p<0.001)$; II vs III $(p<0.001)$; II vs IV $(p<0.001)$. Las otras comparaciones $p>0.05$. 


\section{Discusion}

El IMC es recomendado por la OMS como un indicador básico para medir estado nutricional. Desde el punto de vista bioestadístico, el empleo del IMC se fundamenta en que cuando el peso (componente de mayor variación de causa ambiental) está ajustado según la talla (componente de menor variación ambiental), el índice resultante y la talla no se correlacionan. ${ }^{8}$ Dicha condición no se cumple en otros índices como la asociación entre peso y talla o la relación peso y talla al cubo. El estado nutricional es un predictor de crecimiento fetal ${ }^{9,10}$ y este hecho proporciona el apoyo a las evaluaciones antropométricas periódicas en la población de embarazadas. Biológicamente, el IMC ha sido considerado una expresión, en términos generales, del crecimiento del producto y los ajustes fisiológicos que experimenta la madre, tales como la expansión del volumen sanguíneo, retención de fluídos y acumulación de tejido graso.11-15 Está además asociado positivamente con la presión diastólica materna. 16 El valor del punto de corte que se utilice para clasificar estado nutricional varía con la edad gestacional. Por esta razón, presentar la evolución del IMC según semanas de amenorrea resulta un aporte valioso para estudios pronósticos. Es evidente que las curvas de IMC independientemente del país de origen muestran un sesgo a la derecha, reflejando un sobrepeso importante en relación al estadísticamente esperado. Este representa el problema nutricional prevalente en las embarazadas. La ciudad de Buenos Aires presenta una importante contribución de población proveniente de otros países del Cono Sur. Las variaciones antropométricas pre gestacionales observadas

\section{Referencias}

1. Sánchez Jaeger A, Del Real Vargas SI, Rodríguez SL, Peña Perdomo E, Barón MA. Índice de masa corporal al comienzo del embarazo en un grupo de gestantes venezolanas de bajo estrato socioeconómico y su relación con la antropometría de sus recién nacidos. Arch Lat Nutr. 2006; 56: $141-5$.

2. Ehrenberg H, Dierker L, Milluzzi C, Mercer B. Low maternal weight, failure to thrive in pregnancy and adverse pregnancy outcomes. Am J Obstet Gynecol. 2003; 189: 1726-30.

3. Bolzan A, Guimarey L, Norry M. Height, weight and body mass index differences between adolescent and adult mothers during pregnancy and fetal growth. Acta Med Auxol. 1999; 31: 9-13.

4. Cnattingius S, Berfstrom R, Lipworth L, Kramer MS: Prepregnancy weight and the risk of adverse pregnancy outcomes. N Engl J Med. 1998; 338: 147-52. podrían reflejar diferencias étnicas, no así el IMC que es más afectado por las costumbres nutricionales y culturales. Si así fuese, el estudio muestra que el IMC pregestacional ajusta para los cuatro grupos de nacionalidad de manera prácticamente igual, aunque haya diferencias en la talla y el peso previo entre algunas de ellas, en tanto que durante la gestación comienzan a diferenciarse los grupos, siendo que se hallaron diferencias significativas en el IMC. Las argentinas mostraron la mayor proporción en ambos sectores extremos de la curva de IMC ya que hubo mayor porcentaje de bajo IMC y de sobrepeso que el resto de las de otras nacionalidades. Por consiguiente podría argüirse un efecto del grupo étnico en el estado nutricional. Asimismo, otro factor determinante fue el nivel educativo, claramente asociado a los valores de IMC y que muestra el efecto del contexto social y de las oportunidades, en este caso de acceso a la educación, sobre un indicador biométrico. Finalmente, un tercer determinante es la edad. Debido a la diferente velocidad de crecimiento del peso y la estatura, el IMC varía con la edad, argumento que explica los distintos valores medios entre este índice en las madres adolescentes y las adultas. Ambas curvas nunca se cruzan a lo largo del embarazo, mostrando claramente dos poblaciones diferentes en cuanto a la evolución de la masa corporal a lo largo de la gestación. Este hallazgo fundamenta la necesidad de realizar una investigación para realizar una curva de referencia de incremento de IMC para embarazadas adolescentes.

En síntesis, la investigación permitió obtener el perfil de estado nutricional durante la gestación empleando curvas nacionales de incremento del IMC ajustado por edad gestacional y observar el efecto de algunos determinantes sociales.
5. Cedergren MI. Maternal morbid obesity and the risk of adverse pregnancy outcome. Obstet Gynecol. 2004, 103: 219-24.

6. Ehrenberg H, Dierker L, Milluzzi C, Mercer B. Low maternal weight, failure to thrive in pregnancy and adverse pregnancy outcomes. Am J Obstet Gynecol. 2003; 189: 1726-30.

7. Calvo EB, Lopez LB, Balmaceda Y, Poy MS, González C, Quintana L, Cámera K, Barrientos E, Berlingueres S, Garciarena S. Reference charts for weight gain and body mass index during preganancy obtained from a healthy cohort. J Matern Fetal Neonatal Med. 2009; 22: 36-42.

8. Cole T. Weight /height $\mathrm{p}$ compared to weight /heoight 2 for assessing adiposity ind childhood: influence of age and bone age on p during puberty. Ann Hum Biol. 1986; 13: 433-51. 
9. Bolzán A, Guimarey L. Relación entre el índice de masa corporal durante la gestación en embarazadas adolescentes y adultas, indicadores de crecimiento fetal y retardo de crecimiento intrauterino. La Costa, Argentina. Arch Lat Nutr. 2001; 51: 145-50.

10. Dietz P, Callaghan W, Cogswell M, Morrow B, Ferre C, Schieve LA. Combined effects of prepregnancy body mass index and weight gain during pregnancy on the risk of preterm delivery. Epidemiol. 2006: 17: 170-77.

11. Wanjiku K, Raynor D. Obstetric outcomes associated with increase in BMI category during pregnancy Am J Obstet Gynecol. 2004; 191: 928-32.

12. Krishnamoorthy U, Schram CMH, Hill SR. Maternal obesity in pregnancy: is time for meaningful research to inform preventive and management strategies? BJOG. 2006, 113: 1134-40.

13. Salinas H, Naranjo B, Valenzuela C, Maisto J, Andrighetti S, Zamorano A, Salinas J, Heuser K. Resultados obstétricos asociados al incremento del índice de masa corporal durante el embarazo en el Hospital Clínico de la Universidad de Chile. Clín Invest Ginecol Obstet. 2008; 35: 202-6.

Recebido em 24 de agosto de 2011

Versão final apresentada em 11 de novembro de 2011

Aprovado em 30 de novembro de 2011
14. Bhattacharya S, Campbell DM, Liston WA, Bhattacharya S Effect of body mass index on pregnancy outcomes in nulliparous women delivering singleton babies. BMC Public Health. 2007; 7: 168.

15. .Sebire NJ, Jolly M, Harris JP, Wadsworth J, Joffe M, Beard RW, Regan L, Robinson S. Maternal obesity and pregnancy outcome: a study of 287,213 pregnancies in London. Int J Obes. 2001, 25: 1175-82.

16. Kiran UTS, Hemmadi S, Bethel J, Evans J. Outcome of pregnancy in a woman with an increased body mass index. BJOG. 2005; 112: 768-72 